\title{
New models for satellite altimeter sea state bias correction developed using global wave model data
}

\author{
N. Tran,1 D. Vandemark,2,3 B. Chapron,4 S. Labroue,1 H. Feng,5 B. Beckley,6 5 and P. \\ Vincent7,8
}

\author{
1 Space Oceanography Division, Collecte Localisation Satellites (CLS), \\ Ramonville Saint-Agne, France. \\ 2 NASA Goddard Space Flight Center, Wallops Island, Virginia, USA. \\ 3 Now at Ocean Process Analysis Laboratory, University of New \\ Hampshire, Durham, New Hampshire, USA. \\ 4 IFREMER/Centre de Brest, Plouzane' France. \\ 5 Center for Atmospheric Sciences, Hampton University, Hampton, \\ Virginia, USA. \\ 6 Raytheon Information Technology and Scientific Services, Landover, \\ Maryland, USA. \\ 7 CNES, Toulouse, France. \\ 8 Now at IFREMER, Issy-les-Moulineaux, France.
}

Adresses

*: Corresponding author : tran@cls.fr

\begin{abstract}
Sea level determination via satellite altimetry is subject to numerous error sources. One of these is the sea state bias where changing surface wave conditions alter an altimeter's estimate of mean sea level. Recent work suggests that present day methods for correcting this bias, based solely on wave and wind information from the altimeter, may be improved if additional surface gravity wave field measurements become available. This paper tests this hypothesis by developing several new sea state bias correction models using a year long combination of Jason-1 data with wave field statistics generated from an hindcast of the WaveWatch3 ocean wave model. Each candidate model is produced in the same manner; through a nonparametric mapping between Jason-1 sea surface height anomaly estimates and two correlatives. The first is always the significant wave height from Jason-1 and the model differences come through choice of the second variable. Past studies dictate our selection of these second parameters and they include terms related to the local wind speed, swell energy, wave age, wave period, and wave slope. Evaluation of model skill is conducted in term of explained variance. Several candidate models do indicate promise for wave model use in future empirical developments. Results show that in the low latitudes the models developed using the swell height and mean period, modestly outperform the operational parameterisation of the bias model. Thus this study indicates that systematic regional error in the present sea level corrections may be improved by inclusion of wave model information.
\end{abstract}

Keywords: sea state bias; altimetry; ocean wave model 


\section{INTRODUCTION}

Satellite altimetry has provided to oceanographers a unique remote-sensing tool for mapping the global ocean topography to study the general ocean circulation, its large-scale lowfrequency variability, turbulent eddy components, and associated transport properties (e.g. Chelton et al, 2001). To be useful, this topography must be measured with an accuracy of a few centimetres. Among the different geophysical processes that affect altimeter measurements, ocean surface waves induce a range bias in the instantaneous retrieved sea surface height $(\mathrm{SSH})$, lowering this measure as much as tens of centimetres below true mean sea level. This error has to be modelled and removed for accurate altimetry measurement of sea level. It is referred to as the sea state bias (SSB) correction.

Thanks to concerted efforts to improve the orbit determination and other geophysical corrections, errors in algorithms once considered of secondary concern such as the SSB correction now become the focus of attention. To date, SSB operational models are developed using empirical relationships between altimeter-observed range bias, wind speed, and significant wave height estimates (Gaspar et al, 1994; Gaspar and Florens, 1998; Gaspar et al, 2002; Labroue et al, 2004); though other closely-related models based on direct power measurements instead of wind speed have also been proposed (Melville et al, 1991; Scharroo and Lillibridge, 2005). Recent consensus suggests that while this approach offers an effective and self-contained (no dependence on external data sources) altimeter correction, this twoparameter SSB model is not entirely accurate since it does not fully parameterize range bias variability attributed to regional complexities in the ocean wave climate (Glazman et al, 1994; Kumar et al, 2003). 
The goal of current research efforts is to explain more of the remaining SSH variability through improved SSB models by including additional measurable or predictable correlatives. Efforts to identify alternative or ancillary SSB model factors have included laboratory, towerbased, and aircraft experiments (Branger et al, 1993; Arnold et al, 1995; Melville et al, 2004, Vandemark et al, 2005), numerical analysis of satellite data (Minster et al, 1992; Glazman et al, 1996), and non-linear sea surface models development (Elfouhaily et al, 2000; 2001; Gommenginger et al, 2003; Millet et al, 2003a; Melville et al, 2004). While models created from satellite data have focused on addressing the degree of wave field development by including a wave age or pseudo wave age parameter to improve the bias estimates ( $\mathrm{Fu}$ and Glazman, 1991; Glazman et al, 1996), analyses derived from field experiment data and different theoretical modelling approaches have rather pointed out the likely impacts due to the presence or absence of swell (Minster et al, 1992; Glazman et al, 1996; Branger et al, 1993) and to wave dynamics involved in tilt modulation, hydrodynamic modulation, and nonGaussian long wave surface statistics (e.g. surface skewness). These latter factors are all associated with higher order moments of the surface height power spectral density (PSD) compared to SWH which is related to the order 0 moment, such as the surface significant slope or wave steepness (Parsons and Miller, 1991; Branger et, 1993) obtained from the second moment of the surface height PSD, the long wave RMS slope (Rodriguez et al, 1992; Chapron et al, 2001; Gommenginger et al, 2003; Millet et al, 2003a; 2003b; Melville et al, 2004; Kumar et al, 2003; Vandemark et al, 2005), the acceleration variance (Elfouhaily et al, $1999 ; 2000 ; 2001)$ related to the fourth moment, the cross-skewness quantity between long wave elevation and slope (Vandemark et al, 2005), or such as finally orbital velocity (Elfouhaily et al, 2001; Chapron et al, 2001; Millet et al, 2003a) related to the first moment of the surface height PSD. 
Through this large diversity of parameters that potentially might represent good SSB correlatives proposed in the literature, we observe considerable disagreement as to which external factors should be accounted for. It is recognized that the task to reach a consensus on which one to recommend is difficult. It is largely because there is insufficient observational information on the space and time characteristics of the non-linear sea surface at the wave scales that are involved in the SSB, since most of these parameters are not readily retrieved from satellite measurements exception of pseudo wave age. To assess the operational value of these potential parameters highlighted through the literature, the dataset that is central to this study is a combined wave model predictions and Jason-1 altimeter measurements set compiled over 2002 year-period. Here the operational wave model, WaveWatch3, is employed. The use of wave model outputs represents a compromise between improving the SSB modelling through better description of the complex local wave climate and assuring possible operational application of such information to each Jason-1 SSH measurement where availability of these independent parameters are required everywhere to support point-bypoint SSB correction.

This dataset enables a number of issues to be examined: first it allows computation of the different external factors identified as potential SSB correlatives everywhere at the altimeter measurement rate. Secondly, the present work provides assessment of alternative 2-parameter SSB models tuned on a global dataset in a manner identical to the operational 2-parameter model in order to discern how, when and where wave model data may contribute to improving the SSB computation in future operational 3 or 4-parameter algorithm development. This objective will be attempted mostly through analysis of the individual effectiveness of each auxiliary parameter in terms of gain in SSH variance reduction.

The paper is arranged as follows: in section 2, we describe the methodology employed to derive the different SSB models as well as the data sources and the data collocation process. 
We also present the chosen correlatives for SSB modelling and the method of SSB model generation. We compare the global and regional effectiveness of each model through rating of their performance in terms of explained variance in section 3. Discussion of the relative significance of the SSB correction upon geographical mean and variance of SSH measurements is provided in section 4 . This analysis carries potential relevance to systematic error in altimetric mean sea surface and mean currents determination. Section 5 concludes this work with discussion on the implications of these results for developing new operational SSB correction.

\section{APPROACH AND DATA}

In this study, we developed SSB models in the form of a regular grid in a two-dimensional space determined by significant wave height and a second variable correlative denoted Pi. We make use of the nonparametric estimation technique (NP) based on kernel smoothing developed by Gaspar et al (1998; 2002). It represents an objective statistical method for developing models that avoids choice of a parametric form that might be controversial and simply assumes that SSB is a (no specified) function of a set of pertinent variables. The resulting form provides a good representation of the actual complexity of the relation between the SSB and its given correlatives. Indeed, this nonparametric estimation technique replaces global polynomial estimate with piecewise of local polynomial approximations. The model development requires choice of a kernel or smoothing function, kernel bandwidth (so that the local polynomials do not depend on data outside their interval of support) and degree of the local polynomial approximation (for details and discussion on choices, see Gaspar et al, 2002). 
Combination of this NP technique with residual sea surface height (RSSH) relative to the mean sea surface GSFC00.1 (Wang, 2001) allows development of SSB models in a simple and straightforward way whatever the two-dimensional space of coordinates used (Vandemark et al, 2002; Labroue et al, 2004; Scharroo and Lillibridge, 2005). Indeed, this direct method works with absolute data, in opposition to methods based on differenced quantities such as crossover or collinear methods (Labroue et al, 2004). It facilitates isolation of the SSB term against the chosen correlatives within the large SSH residuals as more residuals are available than crossovers with moreover better sampling of the large variety of ocean conditions within smaller time period (Vandemark et al, 2002). In summary, the advantage of this approach is that it combines the simplicity and high resolution of the direct method with the NP smoothing technique. Application of the NP technique on SSH residuals was first done by S. Labroue for different altimeters (TOPEX, Geosat Follow-on, Jason-1, and ENVISAT). Model evaluation results can be found for Jason-1 in Labroue et al (2004).

For our purpose, that is the comparison of the impact of different second-order geophysical parameters in the SSB modelling, the model estimation performed with this approach represents a robust way to derive smooth and precise candidate models for SSB estimation with simple implementation and use of reduced number of observations in their development.

\subsection{Data sources and collocated set}

\section{Wave Watch3 wave model}

Global ocean wave modelling has now entered an operational stage, capable of providing full two-dimensional gravity wave spectra everywhere on demand. WaveWatch3 (WW3) is a third version of a wind-wave generation and wave propagation model. It has been developed 
at the National Oceanic and Atmospheric Administration/National Center for Environmental Prediction/National Weather Service (NOAA/NCEP/NWS) in the spirit of the well-known WAM wave model (Tolman, 2002; Tolman et al, 2002). It is a further development of the models WaveWatch1 and WaveWatch2 developed at Delft University of Technology and NASA/Goddard Space Flight Center, respectively. The governing equations simulate temporal and spatial variations of mean water depth and mean current, wave growth and decay resulting from the surface wind forcing, dissipation (e.g. whitecapping), and the effect of the bottom friction on the water column. This NOAA/NCEP implementation of the model is for deep water regions (depth $>300 \mathrm{~m}$ ). Both first- and third-order numerical schemes are used to solve the governing equations forecasting the evolution of the directional wave energy spectra.

WW3 was run for an entire year, 2002, on a global $1^{\circ}$ by $1^{\circ}$ grid over the integration domain from $70^{\circ} \mathrm{S}$ to $70^{\circ} \mathrm{N}$ in latitude and at a 6 hourly time step (Feng et al, 2004; 2005). At each grid point, the spectrum $S$ (f, $\phi)$ of the sea surface elevation $\zeta(\mathrm{x}, \mathrm{y})$ gives the distribution of wave energy among different wave frequencies (f) and directions $(\phi)$ of wave lengths on the sea surface. The spectral resolution is determined by 24 directions and 25 frequencies which are logarithmically spaced from $0.042 \mathrm{~Hz}$ to $0.405 \mathrm{~Hz}$ with intervals of $\Delta \mathrm{f} / \mathrm{f}=0.1$. European Centre for Medium-Range Weather Forecasts (ECMWF) wind fields are used to force the wave model.

Outputs from the model include globally gridded fields of swell and wind sea wave heights (denoted H_swell and H_ws respectively) and numerous wave field statistics. These latter are computed from a given wave height spectrum $S$ as $m_{x}=\iint f^{x} S(f, \phi) d f d \phi$. Parameters $\mathrm{m} 0, \mathrm{~m} 1, \mathrm{~m} 2$, and $\mathrm{m} 4$ in this study represent the wave height variance, the first-order moment, the wave orbital velocity variance and the wave acceleration variance respectively. The different wave model parameters are linearly interpolated in space and time onto the altimeter 
ground track. On a year-period, the wide range of sea surface conditions sampled does provide a unique opportunity to compare the relative robustness of the different SSB derived relationships.

\section{Jason-1 altimeter}

The Jason-1 altimeter was launched on December 2001 and placed on the same ground track as its predecessor TOPEX/Poseidon. Jason-1 carries the Poseidon-2 radar altimeter and occupies a non-sun-synchronous orbit at an altitude of $1336 \mathrm{~km}$ with an inclination of $66^{\circ}$. Poseidon-2 is a dual-frequency radar altimeter that transmits at $13.575 \mathrm{GHz}(\mathrm{Ku}-\mathrm{band})$ and 5.3 GHz (C-band). Detailed description of the mission and the Poseidon-2 instrument are provided by Ménard et al (2003) and Carayon et al (2003). For this study, we use measurements extracted from the Jason-1 GDRs (Geophysical Data Records) over a yearperiod spanning 2002. Selected parameters are the $1-\mathrm{Hz}$ records of significant wave height (SWH), Ku- and C-band normalized radar cross section $(\sigma 0)$, the altimeter-derived wind speed, hereafter called U_alt_Gal and based on both SWH and Ku-band $\sigma 0$ (Gourrion et al, 2002), and finally the altimeter's RSSH. These latter are computed by subtracting the altimeter range measurement from the orbital altitude and correcting for all known atmospheric delays, tides and GSFC00.1 mean sea surface height, expect for the SSB term that we added back onto each residual estimate since it was removed in the residual products that we are using. These RSSHs come from the NASA Ocean Altimeter Pathfinder Project (Koblinski et al, 1998). Interpolated ECMWF surface winds at the measurement location are also provided in the distributed Jason-1 products and this wind product will also be considered as SSB model parameter to test. We adhere to the altimeter quality control 
flagging provided by the NASA/GSFC Pathfinder Project to discard all questionable measurements when generating the dataset.

\section{Collocated set}

WW3 model-estimated wave parameters and Jason-1 altimeter data are collocated by spatial and temporal interpolation onto standard NASA/GSFC altimeter pathfinder nominal ground track locations. There are over 14 million of WW3/Jason-1 data pairs recorded for the 2002 year-period. This study sub-samples the along-track data by taking 1 point every 10 measurements. The performed processing allows good spatial and temporal representation of all regions. The resulting subset is used to develop the different SSB models while the total dataset is used to evaluate the performances of the models via RSSH variance reduction and to assess regional and temporal variability of the SSB estimates.

\subsection{Chosen correlatives for bias correction modelling}

Literature highlights of potential SSB correlatives laid out a very confusing picture as gathered in Table 1 and presented in the introduction section. Performance assessment status of their respective individual effectiveness will be provided in the present analysis to rank them. This parameter list suggests that surface parameters from higher order moment of wave height PSD (wave steepness, RMS slope, wave age) in complement to the leading SWH (related to moment of order 0) dependence may better describe the SSB behaviour than current operational altimeter derived wind speed. Note that the inverse wave age $(\Omega)$ describing the degree of development of the waves and the significant slope or significant wave steepness $(S)$ representing a measure of the mean non-linearity of the surface waves, 
are both related to the long wave orbital velocity variance $(\mathrm{m} 2)$; while the RMS slope representing the short-scale wave slope variance is as for it rather related to $\mathrm{m} 4$ moment of the surface height PSD as defined here. Finally, the two last new ocean wave conditions descriptor that will be tested are the swell height (H_swell) and the mean wave period (Tm) related to the first-order moment $\mathrm{m} 1$, respectively.

Additionally, complementary testing will be provided with use of more common parameters. This is done in an attempt to more clearly delineate the effectiveness of these parameters through consistent model development. In particular, in addition to the two wind speed estimates available in the Jason-1 products (denoted U_ECMWF and U_alt_Gal in Table 1), a third one is computed based solely on Ku-band $\sigma 0$ by making use of the modified CheltonWentz algorithm, MCW, (Witter and Chelton, 1991) adapted to Jason-1 measurements. This wind speed estimate will be referred to as U_alt. This will allow us to examine the effect of the differing but generally similar wind speed products in the context of SSB work that is applicable to all existing altimeter missions. Indeed, Jason-1 mission is the only one among the different missions that uses the multi-parameter algorithm of Gourrion et al (2002). The others - TOPEX, Envisat, and Geosat Follow-on - provide wind speed estimates with adapted MCW algorithms.

An SSB model directly using the Ku-band $\sigma 0$ measurement will also be tested, as will be a Cband version. Indeed, Scharroo and Lillibridge (2005) proposed that all operational altimeters use a non-parametric SSB model in the form of a grid in the two-dimensional space determined by $\mathrm{SWH}$ and $\mathrm{Ku}$-band $\sigma 0$ to bypass issues between sensors related to varied wind speed retrieval methods, modeling forms and parameterizations. The present model intercomparison effort should help to inform the debate on this choice within SSB model formulation. 
Pseudo wave age $(\xi)$ derived from altimeter measurements of SWH and U_alt will be the last parameter to test in this 11-parameter selection list. Past analysis from Fu and Glazman (1991) has attempted to account for the effects of wave type (e.g. fully developed swell versus wind waves) on SSB estimates by the means of this altimeter derived quantity. Updated testing will be possible with the present dataset and its performance results will be compared with those from other proposed parameters to provide the most complete comparison status possible at this time.

Note that if the choice of wave model output parameters for use in SSB studies should be driven by theory and field results, the quality of the wave spectral estimates and derived statistics also requires strong consideration. Expected model smoothing and/or inadequate physics related to higher order wave moments may limit any possible gain to be obtained from wave model outputs. Indeed, our chosen wave model parameters in the WW3 dataset are highly correlated with a correlation coefficient $\mathrm{R}^{2}$ of $0.99,0.94$, and 0.80 between respectively $\mathrm{m} 1, \mathrm{~m} 2, \mathrm{~m} 4$ and $\mathrm{m} 0$ even if a small decreasing trend is observed when increasing the order of the moments. This is partly because they are derived from the same spectrum but also because the wave model does not likely carry all true dynamics of the higher order moments. So choice of optimum parameters to include in new operational SSB model that is driven by both theoretical and field experiment results may be compromised by such limitations. Note moreover that theory based model developments assume ocean conditions in the absence of swell and ocean currents, so the parameters that they advocate can perform not as expected under more realistic ocean conditions. This could be also the case with the ocean conditions encountered during field experiments for which conditions could differ much from the open ocean where wave spectra tend to be much broader than those measured in closed or semiclosed sea basins, such as bays, internal seas and coastal regions. All these considerations 
should be considered within the present analysis, providing an updated state of the art on the SSB framework that blends empirical and theoretical influences.

\subsection{Bias model generation}

In this study, each candidate bias correction model is produced in the same manner; through a nonparametric mapping (Gaspar and Florens, 1998; Gaspar et al, 2002) between Jason-1 RSSH estimates and two correlatives. The first is always the SWH from Jason-1 and the model differences come through choice of the second variable. The derived models are thus in the form of a regular grid in a two-dimensional space and are denoted SSB ( $\mathrm{SWH}, \mathrm{Pi}$ ). Pi is chosen from the list provided in Table 1. The model denoted SSB (SWH, U_alt) will serve as the operational reference (i.e. operational parameterization choice) for comparison; while the single-term model, SSB $(\mathrm{SWH})=-3.8 \% \mathrm{SWH}$, will represent the low limit benchmark. This latter model will serve to evaluate the effectiveness of each second-order parameter in the SSB modelling and to determine the regional contribution of this parameter to the leading dependence on SWH. The first reference model will help to point out changes that the different models tested will bring compared to the actual operational modelling. The choice of not using the Jason-1 operational model but rather a recomputed one with U_alt as reference will provide results that could be generalised to other missions that use more commonly U_alt rather than U_alt_Gal as Jason-1 does. All the models will be developed in a consistent and homogeneous way, i.e. same model estimation process, same applied corrections to compute the RSSHs and same filtering of the anomalous data. This should reduce the number of potential source of discrepancies that are often difficult to separate and evaluate when comparing different model analyses found in the literature. 
Compared to previous developments of empirical models, the results presented in the following will offer us new insights into the SSB behaviour and modelling since they allow discussion on the global relevance of various potential SSB correlatives for satellite altimeter operational use. Up to now, there have been only few attempts to empirically derived new models beyond the usual correlations of SSB with altimeter derived wind speed and SWH. They are based mostly on analyses from tower-based experiment data to take into account surface characteristics such as RMS wave slope and wave age (Millet et al, 2003a; 2003b; Melville et al, 2004). Difficulties to inter-compare the results between previous analyses arise at least from combination of seven factors and makes interpretation of the discrepancies most of the time difficult. Evaluation of the impacts of these different factors is not always feasible outside a given analysis and quantitative comparisons between different empirical modelling based works are sometimes hazardous.

The first source of discrepancy comes from the fact that there are differences between the sea state relating bias measured from satellite and that observed from tower. This latter allows direct measurement of the electromagnetic (EM) bias due to stronger reflection by the wave troughs than by the wave crests which results in a discrepancy between the mean sea surface and the mean reflecting surface. In satellite data, oversimplifications of the algorithms used for the analysis of the altimeter waveforms give rise to two other sea state related biases, the skewness and tracker biases (e.g., Chelton et al, 2001). They are lumped together with the EM bias to form the so-called altimeter SSB that we are analysing. These additional terms make difficult inter-comparison of the in-situ and satellite bias data dependencies on sea surface characteristics. These latter are additionally not derived from same source of measurements due to availability and constraint in operational chain, buoy versus wave model, and thus exhibit different characteristics and uncertainties. 
Most recently, Kumar et al (2003) explored such comparisons. They investigated the applicability of the tower-based Melville et al (2004) parameterisation of the EM bias on wave slope and wave age information to altimeter data. Their results showed that use of measurements of wave fields from buoys or from operational wave model (WAM) data at the few buoy locations, provides EM bias corrections that basically agree but show also significant scatter. These discrepancies come from both estimates of wave slope characteristics from the wind-sea component (Rodriguez et al, 1992, Gommenginger et al, 2003). Indeed, if wave model predictions of the high-frequency tail of the wave spectrum need improvement, on the other hand, direct observations of wave slope are difficult too, since estimates based on time series data are contaminated by the effects of the orbital motion of the longer waves.

The third cause of discrepancy in the list is related to the difficulty to compare empirical derived models due to the very local character of the in-situ derived models compared to global ones as determined in the present study. Indeed, specific regional features of wave conditions and range extend of the wave parameters cannot be simply generalized as global ocean characteristics.

The next source of differences is linked to the fact that tower experiments allow direct measurements of the EM bias (Arnold et al, 1995; Melville et al, 2004). These experiments used a Ku-band altimeter to measure the apparent sea surface height while measurements of the ocean surface height were made using wave gauges. The EM bias represents the difference between the measured and apparent sea surface heights. The metric used, in this context, in determining the most effective models is the RMS value of the residual errors between the estimated bias and the measured truth value. Determination of SSB models based on satellite data relies on variance minimization techniques (Gaspar et al, 1994; Gaspar and Florens, 1998), i.e. minimization of the total SSH variability, globally, in space and time from 
SSH differences at crossover points or along collinear tracks or from RSSHs as done here. This is performed through search of optimal geographical correlations between SSB and wind-wave characteristics that reduced this variability.

Another factor of difference in comparing the different bias corrections is that the different satellite models depend also on the quality of the SSH estimate used to develop them and thus on the quality of the geophysical corrections used to compute SSH and the orbit determination. Indeed, to satisfy evolving requirements to achieve the highest possible data quality, improved algorithms are being continually evaluated and improved by the science team and so use of different version of any corrections might lead to some different impacts in the SSB modelling in term of correlating regional errors for instance.

Finally, the two last but not the least source of difference between empirical models is that most existing ones are least-squares fits of parametric models to wind and sea parameters. Nonparametric estimators have been proposed to overcome a major drawback of the parametric models that do not necessarily represent well the actual complexity of the relation between the SSB term and its chosen correlatives. Gaspar and Florens (1998) and Millet et al (2003) have compared nonparametric SSB models to parametric ones developed on satellite and tower based datasets, respectively. Improvements over parametric models are observed in both studies. Global optimisation of the models may introduce significant regional errors and these latter can be different from one model to the other. Additionally the number of correlatives in the regression model is also a source of discrepancy between models when they are compared in term of explained variance or RMS of residual errors. Since data have more freedom to vary when more correlatives are used, this leads to increase the explained variance by a model compared to another one based on a lower number of variables. This aside discussion should be kept in mind when analysing results from different works and might help to keep things in perspective. 
Presently back on track, details on the method implementation and estimation process used can be found in Gaspar et al $(1998 ; 2002)$ and Labroue et al (2004). Noteworthy this method uses a local tuning of the kernel bandwidth instead of a global one that allows adaptation of the amount of smoothing to the local data density. This is beneficial in the data-sparse regions and will avoid the use of unnecessarily large bandwidths that would mostly degrade the estimation in data-rich regions. The local bandwidth rule that is applied in the twodimensional problem is expressed as a reference bandwidth (h1, h2) weighted by the local data density (Gaspar et al, 2002) with h1 and h2 setting depending individually on the corresponding SSB correlative. To ease the management of the various coordinates in the SSB modelling process, we did fix $\mathrm{h} 1$ to a constant value equal to $\mathrm{h} 2$ that will additionally remains the same whatever the two parameters considered. This would avoid the delicate task of adapting the bandwidth sizes for each algorithm determination. To use such setting we preprocessed the input data. We normalized them by using the respective mean and standard deviation computed from the different parameter distributions. This will allow furthermore use of the same defined regular grid, with equal spacing in the two dimensions whatever the parameters choice and their respective intervals of variation. The two-dimensional domain and so the SSB models are defined by $151 \times 151$ grid points with $0.1 \times 0.1$ spacing. Due to the very large dynamic range of variation of some parameters, we did in some cases use the logarithm values of the parameters before performing the normalization step. This additional processing concerns the pseudo inverse wave age and the wave steepness parameters. Validation on the bandwidth reference choice was performed by comparing different model estimates with simple bin-averaged model grid on different pairing of two-dimensional coordinates to evaluate the NP smoothing; results prove to be very satisfactory. We are now in possession of a very powerful tool for investigating SSB dependence on different parameters. 
The estimated SSB models are determined to within a constant. Therefore once the SSB model is computed, a global shift is applied to respect SSB $(\mathrm{SWH}=0 \mathrm{~m}, \mathrm{Pi}(\mathrm{SWH} 0))=0$. The value $\mathrm{Pi}(\mathrm{SWH} 0)$ represents the value of $\mathrm{Pi}$ for which the measurement population is mainly associated to situations where SWH values are the closest to zero. The exact magnitude differences between models will not be analysed so use of this low limit reference for $\mathrm{Pi}$ is sufficient in this study, i.e. we are interested in the relative regional features of the SSB estimates and not in their respective absolute magnitudes.

To illustrate different SSB models that have been developed to gain insight into how the SSB estimates behave as a function of the new variables introduced in the regression, two examples are shown in Figure 1. They correspond to the bias algorithms based on combination of SWH with swell wave height and mean wave period respectively. Correction values are represented by grids and to help their visualisation, we display isolines of S SB values in the two-dimensional space as commonly done. As can be observed, the magnitude of the SSB estimate is essentially an increasing function with SWH. Variations with the second correlatives are lower and are different from one to the other.

It should be noted that although one of the advantages of nonparametric estimation is in improved modelling of low data-density regions, if the data density is too low then results become spurious even when using local tuning of the kernel bandwidth to locally smooth the results. To indicate regions of highest significance, model plots are shaded into 3 areas where the data density falls into different interval of values. These three zones correspond respectively to cases where the bin-number of data is greater than a threshold of 250 samples and marked by a bin colour in white shade (central area of the plot), in light grey when it is between 50 and up to 250 , and finally in dark grey when there is at least a single sample up to 50. As an aside, this colouring scale helps us to verify the smoothing introduced in the model through the choice of the reference bandwidth and to validate the chosen settings. 
A model feature that can be used to compare the effect of the individual parameter from the different modelling lies in the magnitude of the SSB correction variation, or sensitivity, at a fixed value of SWH such as $2 \mathrm{~m}$. This evaluation is derived within the data rich density zone of each model with a lower threshold limit fixed to 50 samples. Results provide a means to evaluate the potential gain of using alternate parameterizations to explain SSB variability. We expect that improvement in SSB prediction would come when there is greater amplitude in SSB variation for the second correlative. Ranking of the models using this criterion leads to the observation of relatively large value for the mean wave period with a magnitude of change of $4.14 \mathrm{~cm}$. Variability seen using $\mathrm{Ku}$-band radar cross section and the two altimeter wind speed estimates indicates slightly weaker values between 3.2 and $3.7 \mathrm{~cm}$. Large values are also associated to $\Omega$ and $\xi$ with respectively 3.24 and $3.44 \mathrm{~cm}$. All other cases display weak variation of magnitude $(1-2 \mathrm{~cm})$ and among them there is the model based on ECMWF wind speed parameter with the lowest value at $1.2 \mathrm{~cm}$. These evaluations concern only a small sampling of the data population and are therefore indicative but not conclusive of the overall model performance and rating.

\section{VARIANCE REDUCTION RATING}

\subsection{Global evaluation}

Effectiveness of each parameter, from the list gathered in Table 1, is evaluated by examining model performance across the complete 2002 dataset. The common method of rating satellite data based models is to compare the reduction of the total variance in the RSSHs owing to the SSB correction in use. The greater the reduction, the better the model performs globally. 
Figure 2 displays the corresponding explained variance by the different models tested across the global dataset. The reduction of variance achieved by the single-term based model (SSB $(\mathrm{SWH})=-3.8 \% \mathrm{SWH})$ can be viewed as one benchmark (the lowest level) that should be surpassed by any practically useful algorithm. The decrease in RSSH variance through application of this simple SSB term is of $23.9 \mathrm{~cm}^{2}$ globally. Conversely, the model based on the actual operational pairing of SWH and U_alt displays the highest reduction with $24.9 \mathrm{~cm}^{2}$. Note that the other self-contained altimeter models (based on radar cross section measurements, other derived wind speed U_alt_Gal, or pseudo wave age) perform similarly to SSB (SWH, U_alt) model and exhibit thus better performance than models based on combining altimeter and wave model parameters, excepting inverse wave age which however does include altimeter-derived U_alt in its derivation. This fact may explain the very good result obtained since it is equivalent to the one obtained for SSB (SWH, U_alt).

The model based on SWH and ECMWF wind speed shows only a slight improvement since the explained variance reaches a low $24.2 \mathrm{~cm}^{2}$, probably due to the smooth estimates from the meteorological model. Similar low improvement is observed with the model based on RMS slope and suggests that either the wave model does not likely carry all true dynamics of the higher order moments or it represents a not so good correlative as previously thought (Millet et al, 2003a; 2003b; Melville et al, 2004). Other models based upon combined altimeter and wave model data exhibit more similar and better performance. They yield a decrease in total variance of about $24.5-24.6 \mathrm{~cm}^{2}$.

\subsection{Zonal averages}

To more clearly picture the gain associated to the inclusion of wave model derived parameters in the SSB models, we compute zonal averages of the variance reduction in RSSH when 
different SSB models are applied. Figure 3 shows the variance explained by both benchmarks models: SSB (SWH) and SSB (SWH, U_alt) within bands of $10^{\circ}$ of latitude. The nominal RSSH variances have been computed in each band without correcting for the SSB. Then the variances are recomputed on RSSH quantities corrected with the two reference SSB corrections, respectively. The two models introduce similar latitude variation features with minimum explained variance of $\sim 5 \mathrm{~cm}^{2}$ in the equatorial band while maximum values are observed around $45^{\circ}$ of latitudes in both hemispheres and reach $\sim 30 \mathrm{~cm}^{2}$ in the southern band when it is up to $42 \mathrm{~cm}^{2}$ in the northern area. SSB (SWH, U_alt) helps to explain slightly more of the RSSH variance at most latitudes. As discussed by Gaspar et al (1994), the very small explained variance observed in the equatorial ocean is related to the very small variance of SWH. Indeed between $0^{\circ} \mathrm{S}$ and $10^{\circ} \mathrm{S}$, this latter is about $0.2 \mathrm{~m}^{2}$, so the SSB of $-3.8 \%$ of SWH can thus explain a maximum variance of about $4 \mathrm{~cm}^{2}$ as observed.

To facilitate the comparison between the different 2-parameter SSB models against both chosen references, we compute for each latitude band the difference in variance explained by each alternative 2-parameter model from that obtained with SSB (SWH) respectively, as illustrated in Figure 4. Results for the models based on U_alt_Gal and $\sigma 0$ (Ku and $\mathrm{C}$ band) are not shown since they are very close to the one observed when using U_alt. Positive gains relative to the variance reduction observed with SSB (SWH) model are obtained everywhere except for the latitude band centered at $15^{\circ} \mathrm{N}$. As clearly seen, the model based on U_alt improves the explained variance found with SSB (SWH) mostly at high latitudes, greater than $20^{\circ}$ in regions of usually high sea states, with gain larger than $1 \mathrm{~cm}^{2}$; in contrast the improvement is rather small in the tropics. Concerning the two models based on H_swell and Tm respectively, their associated gain in RSSH variance reduction display similar variation with latitudes. These features are clearly reversed from the ones observed with U_alt. We thus obtain an interesting positive impact of these wave model outputs in the tropical area between 
$10^{\circ} \mathrm{N}$ and $20^{\circ} \mathrm{S}$. The variance gain is up to $1.5 \mathrm{~cm}^{2}$ when compared to $\mathrm{SSB}(\mathrm{SWH})$ and this represents a positive gain of about $1 \mathrm{~cm}^{2}$ when compared with the variance explained by the operational parameterization. If the $1 \mathrm{~cm}^{2}$ gain improves the explained variance of $\sim 3.3 \%$ at about $40^{\circ} \mathrm{S}$ with application of the model based on U_alt, this same difference represents rather a large improvement of $\sim 20 \%$ around $5^{\circ} \mathrm{S}$ of latitude when we applied the model based on H_swell. Note that the model based on $\Omega$ exhibits more homogeneous variance reduction gain along latitude besides the deep at $15^{\circ} \mathrm{N}$ which is, however, displayed whatever the model considered. Models based on S and RMS slope display similar variation with latitude with a 3-bump features but overall performance ranking puts the effectiveness of this two ocean condition descriptors behind the gain obtained with either H_swell or Tm in the tropical regions and with U_alt at high latitudes.

The altimeter based or combined parameters based models illustrate different complementary contributions to the basic SWH effects on SSB corrections. The altimeter derived-parameters $\left(\mathrm{Ku}-\sigma 0, \mathrm{C}-\sigma 0, \mathrm{U} \_a l t, \mathrm{U} \_\right.$alt_Gal and $\left.\xi\right)$ bring additional information compared to the SWH dependence SSB term principally at high latitudes while the wave model derived parameters (more particularly $\mathrm{H} \_$swell and Tm) bring significant complementary information on the sea surface wave field, into the SSB correction in the tropical regions.

\subsection{Spatial analysis of the 3 models that stand out}

To better define regional improvement exhibited by the 3 models that stand out in the present test list, we compute maps of variance gain by comparing successively the explained variance obtained by the SSB (SWH, U_alt), SSB (SWH, H_swell), and SSB (SWH, Tm) models respectively with the variance explained by the SSB (SWH) model. This is done in boxes of $30^{\circ}$-longitude by $20^{\circ}$-latitude. Results are provided in Figures 5 and 6 . Indication of the model 
performance is provided in terms of percentage inside each box but also in term of rank between the three models through the box colour. Note that the model based on $\Omega$ modestly outperforms the one based on U_alt in the Pacific area between $40^{\circ} \mathrm{S}$ and $40^{\circ} \mathrm{N}$ with a slightly better gain in variance reduction of $\sim 1.23 \mathrm{~cm}^{2}$ instead of $1.15 \mathrm{~cm}^{2}$ when respectively compared to the variance reduction obtained with the benchmark model SSB (SWH) that is of $10.80 \mathrm{~cm}^{2}$ over this area. We did evaluate the ranking obtained by the models based on $\mathrm{S}$ and RMS slope in term of regional performance; these different models do outperform the 3 selected models in only a rare number of boxes, and are therefore not shown. As visualized in Figure 5, the current operational parameterization displays lower performance in 6 different well defined areas: Mediterranean Sea, tropical Indian Ocean, tropical Atlantic Ocean, tropical western Pacific Ocean, north-western Atlantic ocean, and finally in north eastern Pacific Ocean to cite them. In these areas, the best performer is the model based on H_swell for the Indian Ocean region while for the other ones, it is the model developed with Tm that performs the best amongst the 3 models in comparison, as shown in Figure 6. Table 2 summarizes the relative variance gains that are obtained (in $\mathrm{cm}^{2}$ and in percentage) for the different basins. Variance of respectively, uncorrected RSSHs and RSSHs corrected with the single-parameter model are also provided over the same areas. We observe a 4.70 and $6.25 \%$ gain in explained variance with $\mathrm{H} \_$swell and Tm respectively, while it is only up to $2.33 \%$ with U_alt in the Mediterranean Sea region. In the Tropical Atlantic Ocean, the overall gain obtained with Tm parameterization is of $5.40 \%$ while it is only of $2.37 \%$ with wind speed. The most impressive improvement lies in the tropical Indian Ocean where the gain reaches $35.27 \%$ when using $\mathrm{H} \_$swell while it is shown that the wind speed based model performs worst than the simple SSB (SWH) model with a negative gain of $-3.65 \%$. The performance assessment analysis presented here shows that through the rating and ranking of the effectiveness of the different parameter tested, H_swell and Tm parameters stand out as very 
good candidates to include in 3-parameter even 4-parameter based SSB model in addition to SWH and U_alt in future SSB model development. Their addition as auxiliary correlatives would help to reduce unresolved regional bias errors that might be misinterpreted as seasonal, inter-annual or secular signals in actual SSH data corrected with current SSB parameterization.

In order to better understand the differences in variance reduction obtained by each of these three 2-parameter models, analysis of the relative significance of the SSB term on the geographical mean and variance of SSH measurements, is discussed in the next section. Consequences of these differences of actions may be important for mean sea surface and mean current determination. This comparison is performed by analysing the spatial characteristics of the different SSB estimates and their variability compared to the benchmark represented by the simple SSB (SWH) model.

\section{SPATIAL-TIME VARYING CHARACTERISTICS OF THE SELECTED SSB ESTIMATES}

The differences between the three selected 2-parameter models and the single-parameter one are provided in term of differences of mean corrections and differences of their respective variances in Figures 7 and 8 respectively. In Figure 7, maps of differences are displayed with the same colour scale which represents an interval of variation of $1.2 \mathrm{~cm}$. The magnitude difference is not analyzed since we know that the absolute level of all the estimates is undetermined. Moreover, a geographical uniform mean difference would have no effect on geostrophic velocities estimated from altimeter data. It is, in fact, removed along with all other time-invariant contributions to the sea level estimate. In regions of strong gradients of the differences, the two compared SSB corrections would lead to different estimations of SSH 
leading to distinct estimations of mean geostrophic velocity fields as pointed out by Chelton (1994). As for temporal variability studies, only the differences in variances of the corrections shown in Figure 8 are important.

The different panels in Figure 7 show different behaviours between models with well-defined regions of minimum and maximum differences. It is evident that the two-parameter corrections are consistently larger in magnitude than the single-parameter one (resulting in negative differences everywhere, since both SSB estimates are negative). Some similar regional patterns are found in the different panels associated to each one of the 2-parameter models, with however different extension of the features and relative amplitudes. In the tropical oceans, we observed strong gradients in the grid of difference of mean SSB fields. They coincide with regions of strong gradients in the wind field related to Trade winds belts. These features are also related to sea surface features since the major ocean currents owe their existence to the wind patterns. Surface water just north of the equator is blown from east to west as a function of the Trade winds and corresponds to the Equatorial Counter Current flow, with on either side, strong North and South Equatorial Currents flowing westwards. These three strong zonal flows interact differently with the wave fields and so it is not surprising that their spatial features reflect in the SSB correction behaviour. Indeed, wave-current interactions can locally significantly modify the propagation direction, the shape and the slope of the surface waves and so the SSB values.

For the two combined models: SSB (SWH, H_swell) and SSB (SWH, Tm), the contrast between regions showing minimum and maximum amplitudes of differences, in Figures $7 \mathrm{~b}$ and $7 \mathrm{c}$ respectively, closely matches the zonal characteristics of the correlative tested (Figures not shown) and displays east-west asymmetry within the different basins. Larger negative values are observed in the western coasts of the oceans and smaller ones in the eastern boundaries. Dominant features in global climatology of swell and wind-wave zones, display 
similarly a clear contrast between the eastern and the western boundaries of major basins. It is observed that well-defined zones of swell dominance are located in the eastern tropical areas of the Pacific, the Atlantic, and the Indian Oceans; while regions of intensive wave growth are observed in the northwest Pacific (including the Japan Sea and a large part of the China Seas), the northwest Atlantic, and additionally in the Southern Ocean and the Mediterranean Sea (Young, 1999; Chen et al, 2002). As mentioned, the zonal extension of the swell and wave growth areas presents little similarity with the meridional variation of both wind speed and significant wave height parameters with extreme conditions occurring at high latitudes. The east-west gradients, observed on these maps of differences, seem to reflect a surface characteristic contrast principally located at the boundaries of the ocean gyres. Indeed, maximum horizontal current speeds in ocean gyres are found in the western boundary currents (where instantaneously they can reach $1 \mathrm{~m} / \mathrm{s}$ ). Elsewhere, the currents are substantially weaker with typically speeds of $5-10 \mathrm{~cm} / \mathrm{s}$ except in the tropical and circumpolar belts.

The patterns of large negative differences look well correlated with regions of strong flows and complex wave-current interactions exception of the Agulhas current area. These structures are located in the neighbourhood of the Somali Current in the Arabian Sea, in the Western North Pacific area where there are the Oyashio Current running south and the north flowing Kuroshio Current, in the Tasman basin where there is the strong East Australian Current, and off the east coast of New Zealand where the upstream flow from the Antarctic Circumpolar Current collides with the poleward branch of the South Pacific subtropical gyre circulation. In the Atlantic Ocean, these features are located in three areas: off the east coasts of South America where the weak western boundary Brazil Current encounters the Malvinas Current in one of the most energetic regions in all the oceans, in the Western North area in the vicinity of the southward Labrador Current and poleward Gulf Stream. The equatorial Atlantic is as for it a complex region with a narrow geometry of the basin. The surface 
currents in its western region show a westward flowing North Equatorial Current and a westward flowing South Equatorial Current. The coastal and near-coastal region along the east coast of South America is, as described by Arnault (1987) and others, a spawning ground for meso-scale eddy activity, which compounds the difficulties in discerning the general flow regimes.

Observations of these large SSB dynamics, from estimates based on H_swell and Tm as second order correlatives, in the neighbourhood of western boundary currents but not in the Agulhas current vicinity is, in our point of view, because of the limited fetch at the western boundaries. The wave model seems to definitely capture that spatial characteristic in H_swell and Tm but due to the absence of land mass directly to its west, the Agulhas region does not share these shorter fetch characteristics and does not show up in term of large SSB difference compared to model estimates based only on SWH.

The eastern coasts of the basins show mostly lower negative differences. These regions are characterized by much weaker current flows but also by presence of large swells originally generated in the intense wave regions in both hemispheres and in particular of the Southern Ocean between Australia and South Africa and that propagate northward and contribute to well defined swell pools located particularly off the west coasts of the Americas as well as Australia and weaker ones in the Atlantic Ocean compared to the areas in the Pacific and Indian Oceans (Young 1999).

Parameterization of the SSB term with SWH, U_alt, H_swell, and Tm seems to provide access to different wave information, primarily the changing level of surface wave nonlinearities. Results delineate qualitatively errors in the current parameterisation of the operational SSB correction attributed to regions of swell, growing seas, and boundary currents. They suggest that the bias correction can be significantly improved through a combined use of altimetry and wave model data. 
Concerning the time varying correction, maps of differences are provided in Figure 8. Over much of the world ocean, the variance differences between the models are small between -1 and $2 \mathrm{~cm}^{2}$. The 2-parameter models display local decreases (larger than $3 \mathrm{~cm}^{2}$ in magnitude) in variability in the high-latitude regions of high wind speed and wave height from both hemispheres compared to the SWH linear dependence model result. Patches of local increase (larger than $3 \mathrm{~cm}^{2}$ ) are less well defined except in the Arabian Sea.

Panels (b) and (c) of Figure 8 show similar features than the panel (a) of the same Figure, western coasts of Pacific and Atlantic oceans in the Northern hemisphere and Mediterranean Sea excepted where the combined parameter based SSB estimates are locally characterized by larger temporal variability than those computed with solely SWH. Tropical areas are characterized by a relatively small variability of the SSB correction compared to high-latitude regions, reflecting a moderate level of ocean dynamics. Regions of western currents are more active as only indicated by the variance of the SSB estimates provided by the combined altimeter-wave model based models using H_swell and Tm.

As illustrated, even if the global reduction in variance of the SSH residuals is used as a measure of the SSB model performance to rate the different proposed algorithm, it should not be the unique criterion to take into account to choose a model over another. As shown here significant differences in performance can be observed for particular areas in which local wave conditions differ from the global average and are not well represented by the current operational SSB parameterization while only marginal differences are observed in the global variance reduction evaluations.

Observation of geographical gradients on grid of difference of mean SSB correction fields in Figure 7 and the fact that there are significant differences in variability of the SSB estimates reported in Figure 8, it can be concluded that the different 2-parameter models would have significant different regional impacts when used for studies of both the mean and time- 
varying ocean circulation. Geostrophic velocities are computed from sea level gradients derived from altimeter SSH analyses (Bonjean and Lagerloef, 2002). Improvement in the accuracy of the SSH estimates through better SSB modelling in regions of energetic currents would provide valuable additional insight into the quantification of these flows (in term of variations about the mean) and in the local mean sea surface height description which takes into account the mean ocean currents along with the geoid influence.

Quantification of the precision of the different SSB estimates in different regions is out of the scope of the present work and arise the fundamental problem of finding a measure of goodness to evaluate the uncertainties of the SSB estimates from different algorithms. Nowadays field measurements are too limited and to address this issue, one needs to have both in-situ wave and SSH measurements associated to their satellite equivalents along with wave model data available at the same location and over a long period of time. Moreover such datasets need to sample several dynamically distinct regions with enough variability in the sea state conditions to allow derivation of global model and are not yet thoroughly available.

The altimeter based and combined parameters based models exhibit different features complementing the basic SWH effects on SSB corrections. The altimeter derived-wind speed bring additional information compared to the SWH dependence of the SSB term principally at high latitudes while the wave model derived parameters (H_swell and Tm) bring significant complementary information on the sea surface wave field, into the SSB correction in Mediterranean sea, tropical Atlantic and Indian Oceans and at the boundaries of the ocean basins. These results suggest that a pragmatic path to improve SSB modelling would rely on a 3-parameter even 4-parameter model combining altimeter and wave model outputs as correlatives.

\section{CONCLUSION}


To improve actual SSB models depending only on altimeter SWH and wind speed, additional parameters must include complementary sea surface description information. Indeed the ocean is a complex system and its characterization by these two parameters solely represents a crude and first-order approximation of the real behaviour of its ever changing surface even if they are effective in term of performance assessment with the common measure of goodness (explained variance) used to rate it. Use of wave model outputs in operational SSB algorithm helps to overrun the major obstacle raised by insufficient sampling of additional sea surface parameters to be included in the sea state correction and thus offers exciting new possibilities. Some of them, from the test list, appear to be effective and efficient in characterizing particular aspects of the SSB behaviour. This work was motivated by the hypothesis that better ocean wave condition description will result in improved algorithm solutions.

Combination of the direct method (Vandemark et al, 2002), that works directly with the RSSHs relative to a given mean sea surface and any given correlatives, with a non-parametric estimation technique (Gaspar and Florens, 1998) allows one to readily statistically map the SSB bias. It represents a simple and useful tool employed in the present analysis to test alternative parameterisation of the bias. We evaluated the potential dependence of the bias upon different correlatives and compared these new algorithms with the first order development (linear dependence on SWH) model to assess their individual effectiveness in term of variance reduction and also through the characterization of their geographical impact. As already pointed out in previous analyses (e.g. Glazman et al, 1994), it is one thing to reduce the overall SSH residual variance by finding better descriptors to correlate to the SSH residuals but focus needs also to be on the geographical impact of these different models and their spatial and temporal variability. These latter are of primary concern for scientific applications since altimetric measurements must be corrected for the SSB accurately 
everywhere and actual operational models do not faithfully represent the overall complex SSB.

From this work, it appears that the differences between the SSB algorithms are relatively small when compared at a global scale. What the results also point out is that certain wave model statistics (swell wave height and mean wave period in particular) can yield a difference in SSB correction from that seen using altimeter wind speed, and that a new regional picture of the SSB behaviour does emerge along with a significant variance reduction in the tropical area when compared with the current operational modelling as summarized in Table 2 . The two alternative models, that stand out, are shown to qualitatively retain moreover a pertinent east-west asymmetry characteristic that is related to either wave-current interactions which are particularly pronounced in term of variability in the northern hemisphere where all zonal flows are blocked and deviated by coasts and/or the spatial pattern of dominant swell and wind wave zones which corresponds to these same areas.

This work shows therefore that regional differences may be large and a full exploitation will require an improved fundamental understanding of the SSB and its correlation with oceanographic variables. Major differences between altimeter based models and combined altimeter and wave model parameter based models occur primarily in the tropical regions and at the boundaries of the ocean basins, regions where the actual operational models are suggested to present lower accuracy compared to open ocean with actual knowledge on the SSB dynamics and theory. In contrast, there is an increasing demand for improved accuracy due to the wide use of these regions along our populated coastlines.

There are still questions about the precision and accuracy of both wave model predictions and satellite observations of SSH. In other side, full understandings of the interaction of the EM pulse with the surface wave field, the interaction of the wave field with the flow field, the impact of the wind forcing on both SSH and SWH measurements, skewness and tracker 
biases dependence upon sea state are still lacking. All these issues are presently under investigation but no clear pictures are yet available.

From this work, it appears that the sea state parameters that stand out in the present analysis (swell height and mean wave period), are not the ones expected (RMS slope, wave steepness and wave age) from field experiment and theoretical results. Though past satellite study based on swell height (Minster et al, 1992) showed that SSB dependence upon swell height is small compared to its dependence on wind wave when analysing a few cases of surface front of SWH. This result is in good agreement with the contrast observed here. Concerning Tm effects, there has been no such analysis of SSB dependence on mean wave period.

Note that the comparison of empirical models based respectively on tower experiment data and satellite ones are most of the time uneasy. Indeed, in-situ models directly correlate EM bias measurements to given sea surface parameters by reduction of RMS errors between model and measured data; while satellite model extract SSB signal as a function of given correlatives through RSSH variance minimization.

Though, the aim of current research efforts is thus to reduce variability in either RMS errors or RSSHs by including additional or better descriptor parameters in models, inter-comparison results of EM bias dependence with SSB one should be interpreted with caution due to the fact that these two quantities are not equivalent and additionally the two approaches (in-situ and satellite based) are developed from different sources of surface wave information, buoy versus satellite derived or wave model. Each one of them has their own characteristics and uncertainties, as well as differences in extend in their respective interval of variation. So all these parameter differences could also give rise to different behaviours when correlated to sea-state related bias. Cross-checking comparisons of dependences within a same analysis are keenly encouraged since they would draw a clearer picture on discrepancies and similarities. 
To conclude, in brief even if wave model predictions still present some flaws, indeed it is suggested here that the moments of order 0 and 1 related to wave height and period computed from WW3 wave model provide better useful information for SSB modelling than higher moments ( $\mathrm{m} 2$ and $\mathrm{m} 4$ ) related to wave steepness, wave age, and RMS slope. It is beyond question that it brings howsoever new highlights in the SSB modelling in reducing some regional error impact of this correction on the SSH measurements. Even attempts to use the derived pseudo wave age parameter from altimeter data (as re-done here) does not work as well as introducing into the SSB algorithms information provided from the wave model. The present results demonstrate the usefulness of this source of data to improve the accuracy of SSH restitution. Qualitative assessment has been provided through the present analysis; future works should focus on determination of means to quantify such error reduction. The outcome of such studies will be especially important for the dynamical interpretation of ocean observations and for improvement of present ocean general circulation models.

Given the reasonable results obtained in this study and their general consistency with common general knowledge on wave climate and interactions with both swell and currents, further exploration based on 3-parameter even 4-parameter modelling of the SSB correction, by combining altimeter and wave model derived parameters, will undoubtedly lead to a more realistic and more complete description of the SSB behaviour through an optimal multivariate fusion algorithm determination. 


\section{REFERENCES}

Arnault, S., Tropical Atlantic geostrophic currents and ship drifts, J. Geophys. Res., 92(C5), 5076-5088, 1987.

Arnold, D. V., W. K. Melville, R. H. Stewart, J. A. Kong, W. C. Keller, and E. Lamarre, Measurements of electromagnetic bias at Ku and C bands, J. Geophys. Res., 100(C1), 969980, 1995.

Bonjean, F., and G.S.E. Lagerloef, Diagnostic model and analysis of the surface currents in the tropical Pacific ocean, Journal of Physical Oceanography, 32, 2938-2954, 2002.

Branger, H., A. Ramamonjarisoa, and L. Bliven, A Ku-Band laboratory experiment on the electromagnetic bias, IEEE, Transactions on Geoscience and Remote Sensing, 99(C6), 12,581-12,591, 1993.

Carayon G., N. Steunou, J.-L. Courriere, and P. Thibaut, Poseidon-2 radar altimeter design and results of in-flight performances, Mar. Geod., 26, 156-165, 2003.

Carrère, L. and F. Lyard, Modeling the barotropic response of the global ocean to atmospheric wind and pressure forcing - Comparison with observations, Geophys. Res. Lett., 30(6), 1275, 2003. 
Chapron, B., D. Vandemark, T. Elfouhaily, D. R. Thompson, P. Gaspar, and S. Labroue, Altimeter sea state bias: A new look at global range error estimates, Geophys. Res. Lett., 28(20), 3947-3950, 2001.

Chelton, D. B., J. C. Ries, B. J. Haines, L.-L. Fu, and P. S. Callahan, Satellite altimetry, in Satellite altimetry and Earth Sciences, Ed. Fu L. and Cazenave A., Int. Geophys. Series, 69, 1$131,2001$.

Chelton, D. B., The sea state bias in altimeter estimates of sea level from collinear analysis of TOPEX data, J. Geophys. Res, 99, 24,995-25,008, 1994.

Chen G., B. Chapron, R. Ezraty, and D. Vandemark, A global view of swell and wind sea climate in the ocean by satellite altimeter and scatterometer, J. Atmos. Oceanic Technol., 19, 1849-1859, 2002.

Elfouhaily, T., D. R. Thompson, B. Chapron, and D. Vandemark, Improved electromagnetic bias theory: Inclusion of hydrodynamic modulations, J. Geophys. Res., 106, 4655-4664, 2001.

Elfouhaily, T., D. R. Thompson, B. Chapron, and D. Vandemark, Improved electromagnetic bias theory, J. Geophys. Res., 105, 1299-1310, 2000.

Elfouhaily T., D. R. Thompson, D. Vandemark, and B. Chapron, Weakly nonlinear theory and sea state bias estimations, J. Geophys. Res., 104, 7641-7647, 1999. 
Feng, H., D. Vandemark, B. Chapron, and B. Beckley, Use of a global wave model to correct altimeter sea level estimates, in Proceeding: IEEE International and Geoscience and Remote Sensing Symposium, Anchorage, Alaska, 2004.

Feng, H., D. Vandemark, Y. Quilfen, B. Chapron, and B. Beckley, Assessment of wind forcing impact on a global wind-wave model using the TOPEX altimeter, submitted to Ocean Engineering, 2005.

Fu, L., and R. Glazman, The effect of the degree of wave development on the sea state bias in radar altimetry measurement, J. Geophys. Res., 96, 829-834, 1991.

Gaspar, P., and J.-P. Florens, Estimation of the sea state bias in radar altimeter measurements of sea level: Results from a new nonparametric method, J. Geophys. Res.,103, 15,803-15,814, 1998.

Gaspar, P., F. Ogor, P.-Y. LeTraon, and O.-Z. Zanife, Estimating the sea state bias of the TOPEX and POSEIDON altimeters from crossover differences, J. Geophys. Res., 99, 24,98124,994, 1994.

Gaspar, P., S. Labroue, F. Ogor, G. Lafitte, L. Marchal, and M. Rafanel, Improving nonparametric estimates of the sea state bias in radar altimetry measurements of sea level, J. Atmos. Oceanic Technol., 19, 1690-1707, 2002.

Glazman, R.E., A. Greysukh and V. Zlotnicki, Evaluating models of sea state bias in satellite altimetry, J.Geophys. Res., 99(C6), 12581-12593, 1994. 
Glazman, R., A. Fabrikant, M. Srokosz, Numerical analysis of the sea state bias for satellite altimetry, J. Geophys. Res., 101, 3789-3799, 1996.

Gommenginger, C. P., M. A. Srokosz, J. Wolf, and P. A. E. M. Janssen, An investigation of altimeter sea state bias theories, J. Geophys. Res., 108(C1), 3011, doi:10.1029/2001JC001174, 2003.

Gourrion, J., D. Vandemark, S. Bailey, B. Chapron, C. P. Gommenginger, P. G. Challenor, and M. A. Srokosz, A two-parameter wind speed algorithm for Ku-band altimeters, J. Atmos. Oceanic Technol., 19 (12), 2030-2048, 2002.

Koblinski C.J., B.D. Beckley, R.D. Ray, Y.M. Wang, L. Tsaousi, A. Brenner, and R. Williamson, NASA Ocean Altimeter Project, Report 1, in Data Processing Handbook, NASA Tech. Memo, TM-1998-208605, 1-43, 1998.

Kumar, R., D. Stammer, W. K. Melville, and P. Janssen, Electromagnetic bias estimates based on TOPEX, buoy, and wave model data, J. Geophys. Res., 108(C11), 3351, doi:10.1029/2002JC001525, 2003.

Labroue S., P. Gaspar, J. Dorandeu, O.Z. Zanife, F. Mertz, P. Vincent, and D. Choquet, Nonparametric estimates of the sea state bias for Jason-1 radar altimeter, Mar. Geod., 27, 453-481, 2004. 
Melville, W. K., F. C. Felizardo., P. Matusov, Wave Slope and Wave Age Effects in Measurements of Electromagnetic Bias, J. Geophys. Res., 109(C7), 7018, doi: 10.1029/2002JC001708, 2004

Melville, W. K., R. H. Stewart, W. C. Keller, J. A. Kong, D. V. Arnold, A. T. Jessup, M. R. Loewen, and A. M. Slinn, Measurements of electromagnetic bias in radar altimetry, J. Geophys. Res., 96, 4915-4924, 1991.

Menard Y., L.-L. Fu, P. Escudier, F. Parisot, J. Perbos, P. Vincent, S. Desai, B. Haines, and G. Kunstmann, The Jason-1 mission, Mar. Geod., 26, 131-146, 2003.

Millet, F. W., D. V. Arnold, K. F. Warnick, and J. Smith, Electromagnetic bias estimation using in situ and satellite data: 1. RMS wave slope, J. Geophys. Res., 108(C2), 3040, doi:10.1029/2001JC001095, 2003a.

Millet, F. W., D. V. Arnold, P. Gaspar, K. F. Warnick, and J. Smith, Electromagnetic bias estimation using in situ satellite data: 2, A nonparametric approach, J. Geophys. Res., 108(C2), 3041, doi:10.1029/2001JC001144, 2003b.

Minster, J. F., D. Jourdan, C. Boissier, and P. Midol-Monnet, Estimation of the sea state bias in radar altimeter Geosat data from examination of frontal systems, J. Atmos. Oceanic Technol., 9, 174187, 1992. 
Parsons, C. L. and L. S. Miller, A laboratory study of the electromagnetic bias of rough surface scattering by water water waves, IEEE Trans. Geosci. Remote Sens., 28, 1001-1011, 1990.

Rodriguez, E., Y. Kim, and J. M. Martin, The effect of small-wave modulation on the electromagnetic bias, J. Geophys. Res., 97, 2379-2389, 1992.

Scharroo, R., and J. Lillibridge, Non-parametric sea state bias models and their relevance to sea level change studies, Proc. of the 2004 Envisat \& ERS Symposium, Salzburg, Austria, 2005.

Tolman, H. L., 2002: User manual and system documentation of WAVEWATCH-III version 2.22. Technical Note No. 222, Marine Modeling and Analysis Branch, NCEP, National Weather Service, NOAA, Department of Commerce, 139 pp. [Available at http://polar.wwb.noaa.gov/mmab/papers/tn222/MMAB_222.pdf ].

Tolman, H. L., B. Balasubramaniyan, L. D. Burroughs, D. V. Chalikov, Y. Y. Chao, H. S. Chen, and V. M. Gerald, Development and implementation of wind generated ocean surface wave models at NCEP, Weather and Forecasting, 17, 311-333, 2002.

Vandemark, D., B. Chapron, T. Elfouhaily, and J. W. Campbell, Impact of high frequency waves on the ocean altimeter range bias, J. Geophys. Res., in press, 2005. 
Vandemark, D., N. Tran, B. D. Beckley, B. Chapron, and P. Gaspar, Direct estimation of sea state impacts on radar altimeter sea level measurements. Geophys. Res. Lett., 29(24), 2148. doi :10.1029/2002GL015776, 2002.

Wang, Y. M., GSFC00 mean sea surface, gravity anomaly, and vertical gravity gradient from satellite data, J. Geophys. Res., 106, 31075-31083, 2001.

Witter D.L. and D.B. Chelton, A Geosat altimeter wind speed algorithm and a method for altimeter wind speed algorithm development, J. Geophys. Res., 96, 8853-8860, 1991.

Young, I. R., Seasonal variability of the global ocean wind and wave climate, Int. J. Climatol., 19, 931-950, 1999. 


\section{Table and Figure captions}

Table 1: List of second correlatives tested in complement to SWH as inputs to SSB models.

Table 2: Variance gains (in $\mathrm{cm}^{2}$ and $\%$ ) by comparison of the variance explained by 32 parameter models of SSB respectively with the variance explained by the linear SSB (SWH) model. These 2-parameter models are referenced by their second correlative parameter name.

Figure 1: Sea State bias two-parameter model $(\mathrm{cm})$ based on significant wave height and respectively (a) swell height and (b) mean wave period (shaded areas represent bin data density: (dark gray) from a single sample to 50 ones, (light gray) when it is between 50 and 250 , and (white shade central area) when it is larger than 250 samples).

Figure 2: Reduction in variance performed by the different models on the global 2002 dataset.

Figure 3: Zonal average of the variance explained by the SSB (SWH) and SSB (SWH, U_alt) models.

Figure 4: Zonal average of the variance explained by different models respectively minus the variance explained by the SSB (SWH). Double plots referenced to SSB (SWH, U_alt) serve as benchmarks to compare the different gain in variance between models. 
Figure 5: Maps of regional variance gain (in \%) computed based on the difference of the variance explained by SSB (SWH, U_alt) model and the variance explained by the SSB (SWH) model. They are derived using data binned into $30^{\circ}$-longitude by $20^{\circ}$-latitude boxes. The performances of 3 different 2-parameter models (SSB (SWH, U_alt), SSB (SWH, H_swell) and SSB (SWH, Tm)) are compared with a ranking from 1 to 3. The color of each box represents the performance rank of the SSB (SWH, U_alt) model. Dark gray indicates when this model is the best performer and at the opposite light gray indicates where the model performs worst among the 3 models in competition.

Figure 6: As Figure 5,, results for (a) SSB (SWH, H_swell) model and (b) SSB (SWH, Tm) respectively

Figure 7: Difference of SSB $(\mathrm{cm})$ mean geographical fields computed between estimates from respectively (a) SSB (SWH, U_alt) with U_alt from MCW algorithm, (b) SSB (SWH, H_swell), (c) SSB (SWH, Tm), and SSB (SWH) models. Note that the colour scale displays the same saturated interval of variation of $1.2 \mathrm{~cm}$ centered on the global mean difference for each map.

Figure 8: Difference of SSB variability $\left(\mathrm{cm}^{2}\right)$ geographical fields computed with estimates from respectively (a) SSB (SWH, U_alt) with U_alt from MCW algorithm, (b) SSB (SWH, H_swell), (c) SSB (SWH, Tm), and SSB (SWH) models. 


\section{Tables and Figures}

Table 1: List of second correlatives tested in complement to SWH as inputs to SSB models.

\begin{tabular}{|c|c|c|c|c|}
\hline parameter & symbol & source & definition & references \\
\hline ECMWF wind speed & U_ECMWF & Jason-1 & - & - \\
\hline $\begin{array}{l}\text { Altimeter wind speed from } \\
\text { MCW algorithm (Witter and } \\
\text { Chelton, 1991) }\end{array}$ & U_alt & Jason-1 & $\mathrm{MCW}(\mathrm{Ku} \sigma 0)$ & $\begin{array}{l}\text { Ray and Koblinski (1991), Chelton (1994), } \\
\text { Gaspar et al (1994), Arnold et al (1995), } \\
\text { Gaspar and Florens (1998), Gaspar et al } \\
\text { (2002) }\end{array}$ \\
\hline $\begin{array}{l}\text { Altimeter wind speed from } \\
\text { Gourrion et al (2002) } \\
\text { algorithm }(\mathrm{G})\end{array}$ & U_alt_Gal & Jason-1 & $\mathrm{G}(\mathrm{Ku} \sigma 0, \mathrm{SWH})$ & Labroue et al (2004) \\
\hline Ku-band NRCS & $\mathrm{Ku} \sigma 0$ & Jason-1 & - & $\begin{array}{l}\text { Melville et al (1991), Chapron et al (2001), } \\
\text { Scharroo and Lillibridge (2005) }\end{array}$ \\
\hline C-band NRCS & $\mathrm{C} \sigma 0$ & Jason-1 & - & - \\
\hline Pseudo wave age & $\xi$ & Jason-1 & $3.24\left(\frac{S W H}{U_{-} a l t^{2}}\right)^{0.62}$ & $\begin{array}{l}\text { Fu and Glazman (1991), Minster et al } \\
\text { (1992), Branger et al (1993), Glazman et al } \\
\text { (1996) }\end{array}$ \\
\hline Swell height & H_swell & Wave model & - & - \\
\hline Mean wave period & $\mathrm{Tm}$ & Wave model & $\frac{m 0}{m 1}$ & - \\
\hline Wave steepness & $S$ & Wave model & $\frac{8 \pi}{g} \frac{m 2}{\sqrt{m 0}}$ & $\begin{array}{l}\text { Parsons and Miller (1991), Branger et al } \\
\text { (1993) }\end{array}$ \\
\hline RMS slope & RMS slope & Wave model & $\left(\frac{2 \pi}{g}\right.$ & $\begin{array}{l}\text { Millet et al (2003a), Gommenginger et al } \\
\text { (2003), Melville et al (2004), Branger et al } \\
\text { (1993), Vandemark et al (2005) }\end{array}$ \\
\hline Inverse wave age & $\Omega$ & $\begin{array}{l}\text { Jason-1, } \\
\text { wave model }\end{array}$ & $\frac{2 \pi}{g} U \sqrt{\frac{m 2}{m 0}}$ & Branger et al (1993), Melville et al (2004) \\
\hline
\end{tabular}

Table 2 : Variance gains (in $\mathrm{cm}^{2}$ and $\%$ ) by comparison of the variance explained by 32 -parameter models of SSB respectively with the variance explained by the linear SSB $(\mathrm{SWH})$ model. These 2-parameter models are referenced by their second correlative parameter name.

\begin{tabular}{|c|c|c|c|c|c|c|c|c|c|}
\hline \multirow[b]{2}{*}{ Areas } & \multirow[b]{2}{*}{$\begin{array}{l}\text { Coordinates } \\
\text { (lon } x \text { lat) }\end{array}$} & \multicolumn{2}{|c|}{ Variance of } & \multicolumn{3}{|c|}{$\begin{array}{l}\text { Difference in variance reduction } \\
\text { when compared to the variance } \\
\text { reduction obtained with } \mathrm{SSB}(\mathrm{SWH})\end{array}$} & \multicolumn{3}{|c|}{$\begin{array}{c}\text { Variance gain when compared to } \\
\text { the variance reduction obtained } \\
\text { with } \mathrm{SSB}(\mathrm{SWH})\end{array}$} \\
\hline & & $\begin{array}{c}\text { uncorr } \\
\text { RSSH } \\
\left(\mathrm{cm}^{2}\right) \\
\end{array}$ & $\begin{array}{c}\text { corr RSSH } \\
\text { with } \\
\mathrm{SSB}(\mathrm{SWH}) \\
\left(\mathrm{cm}^{2}\right) \\
\end{array}$ & $\begin{array}{l}\text { U_alt } \\
\left(\mathrm{cm}^{2}\right)\end{array}$ & $\begin{array}{l}\text { H_swell } \\
\left(\mathrm{cm}^{2}\right)\end{array}$ & $\begin{array}{l}\mathrm{Tm} \\
\left(\mathrm{cm}^{2}\right)\end{array}$ & $\begin{array}{c}\text { U_alt } \\
\%\end{array}$ & $\begin{array}{c}\text { H_swell } \\
\%\end{array}$ & $\begin{array}{c}\mathrm{Tm} \\
\% \\
\end{array}$ \\
\hline $\begin{array}{l}\text { Mediterranean } \\
\text { Sea }\end{array}$ & $\begin{array}{l}{[0,30] \times} \\
{[20,60]}\end{array}$ & 109.05 & 14.06 & 0.33 & 0.66 & 0.88 & 2.33 & 4.70 & 6.25 \\
\hline $\begin{array}{l}\text { Tropical Indian } \\
\text { ocean }\end{array}$ & $\begin{array}{l}{[30,90] \times} \\
{[-20,20]}\end{array}$ & 109.18 & 3.59 & -0.13 & 1.27 & - & -3.65 & 35.27 & - \\
\hline $\begin{array}{l}\text { Tropical } \\
\text { Atlantic ocean }\end{array}$ & $\begin{array}{l}{[300,360] \times} \\
{[-20,20]}\end{array}$ & 39.42 & 5.27 & 0.12 & - & 0.28 & 2.37 & - & 5.40 \\
\hline
\end{tabular}


(a)

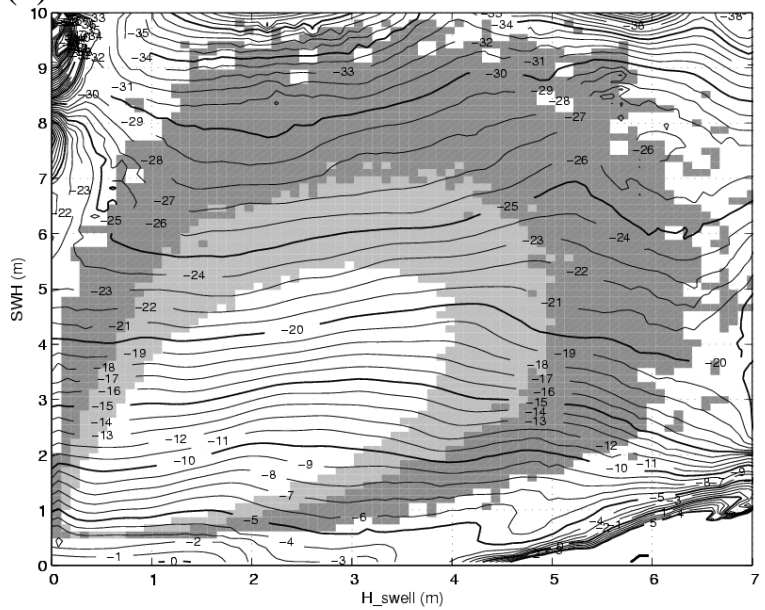

(b)

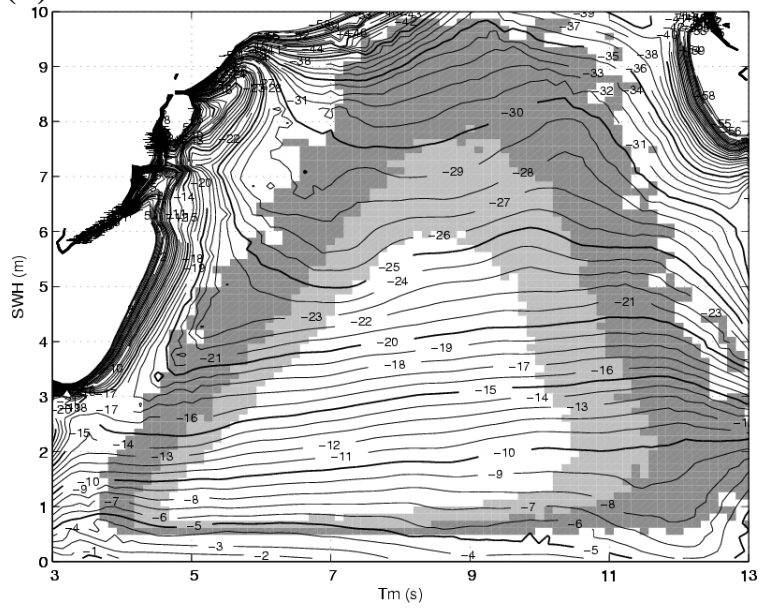

Figure 1: Sea State bias two-parameter model $(\mathrm{cm})$ based on significant wave height and respectively (a) swell height and (b) mean wave period (shaded areas represent bin data density: (dark gray) from a single sample to 50 ones, (light gray) when it is between 50 and 250, and (white shade central area) when it is larger than 250 samples).

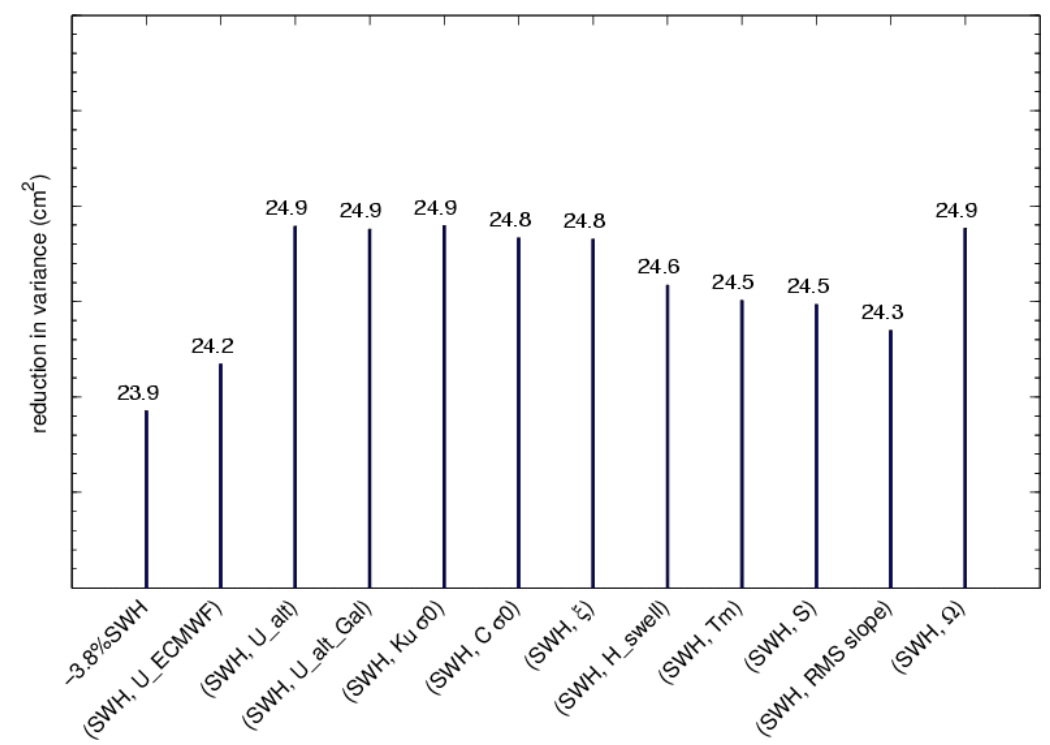

Figure 2: Reduction in variance performed by the different models on the global 2002 dataset. 


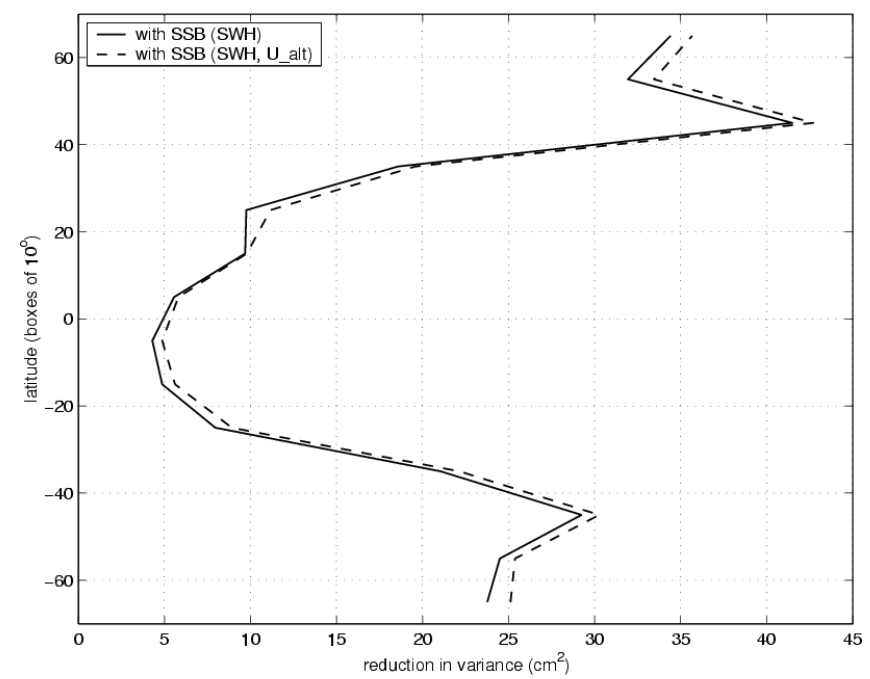

Figure 3: Zonal average of the variance explained by the SSB (SWH) and SSB (SWH, U_alt) models.
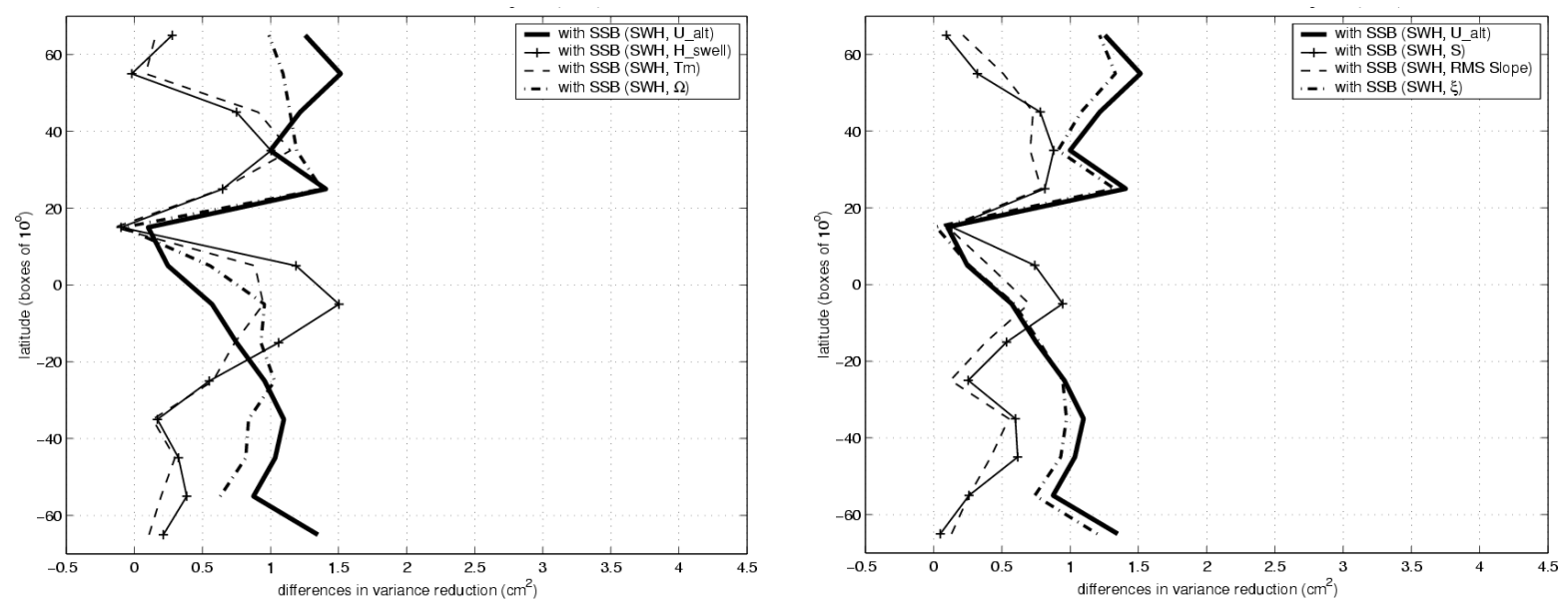

Figure 4: Zonal average of the variance explained by different models respectively minus the variance explained by the SSB (SWH). Double plots referenced to SSB (SWH, U_alt) serve as benchmarks to compare the different gain in variance between models. 


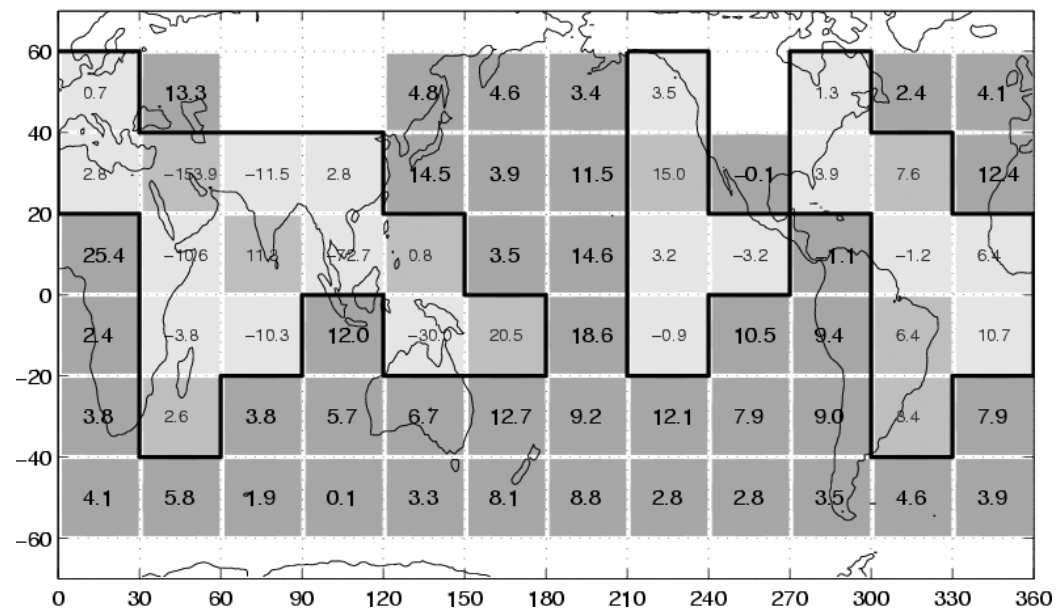

Figure 5: Maps of regional variance gain (in \%) computed based on the difference of the variance explained by SSB (SWH, U_alt) model and the variance explained by the SSB (SWH) model. They are derived using data binned into $30^{\circ}$-longitude by $20^{\circ}$-latitude boxes. The performances of 3 different 2-parameter models (SSB (SWH, U_alt), SSB (SWH, H_swell) and SSB (SWH, Tm)) are compared with a ranking from 1 to 3. The color of each box represents the performance rank of the SSB (SWH, U_alt) model. Dark gray indicates when this model is the best performer and at the opposite light gray indicates where the model performs worst among the 3 models in competition.

(a)

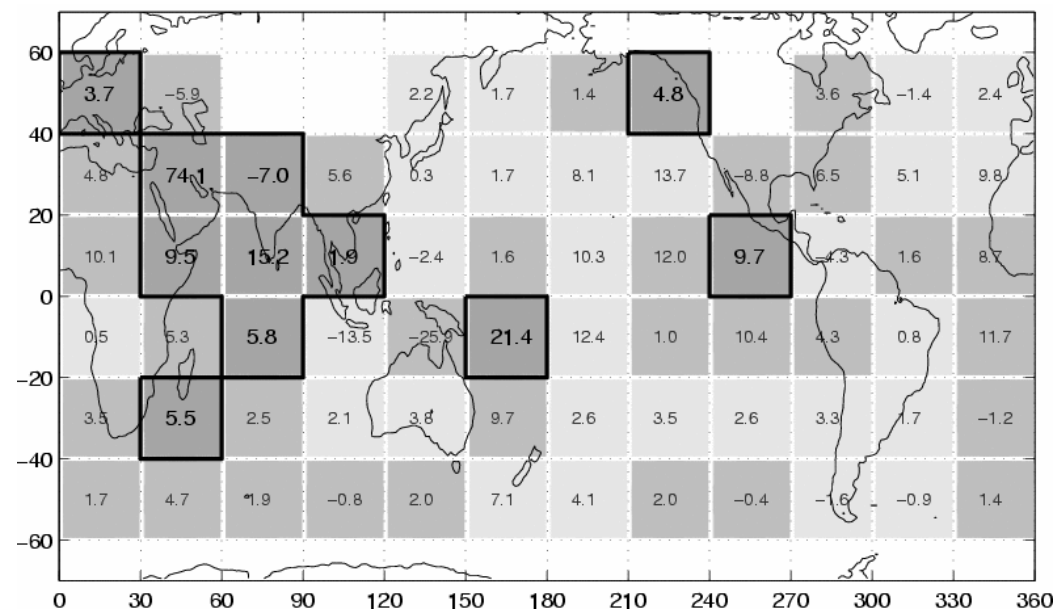

(b)

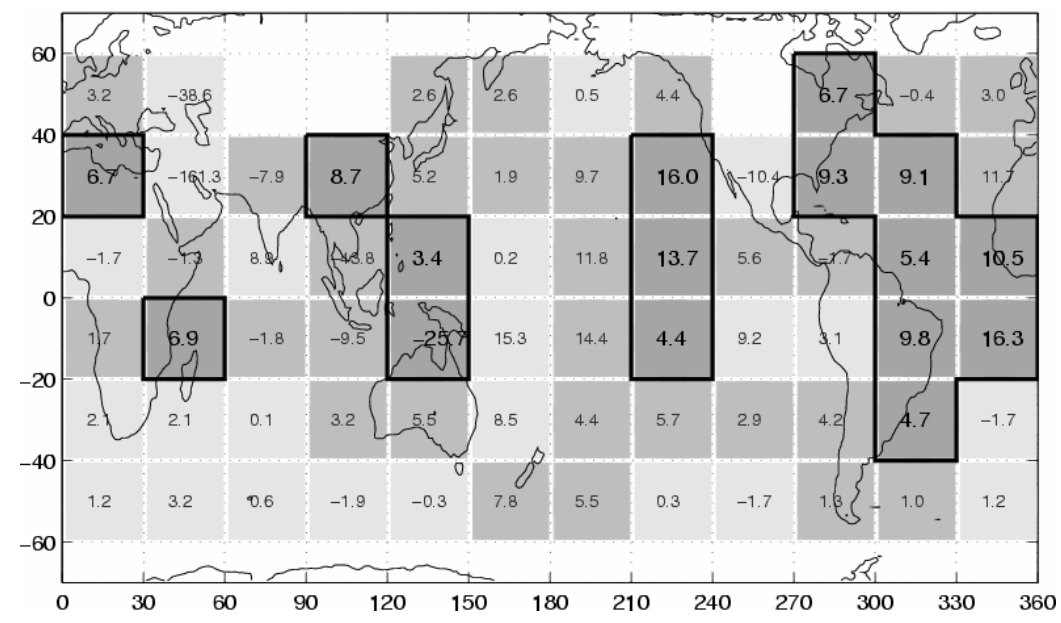

Figure 6: As Figure 5, results for (a) SSB (SWH, H_swell) model and (b) SSB (SWH, Tm) respectively. 
(a)

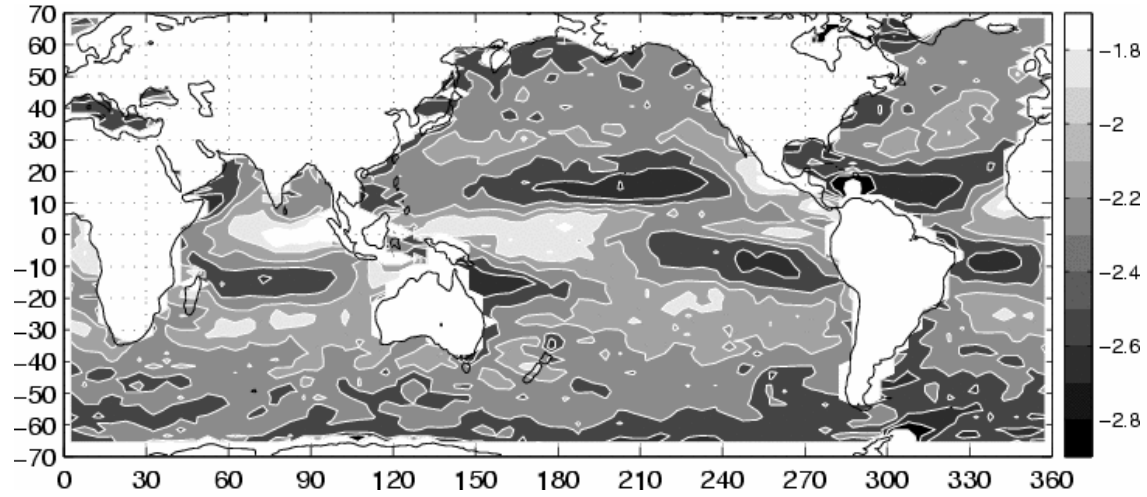

(b)

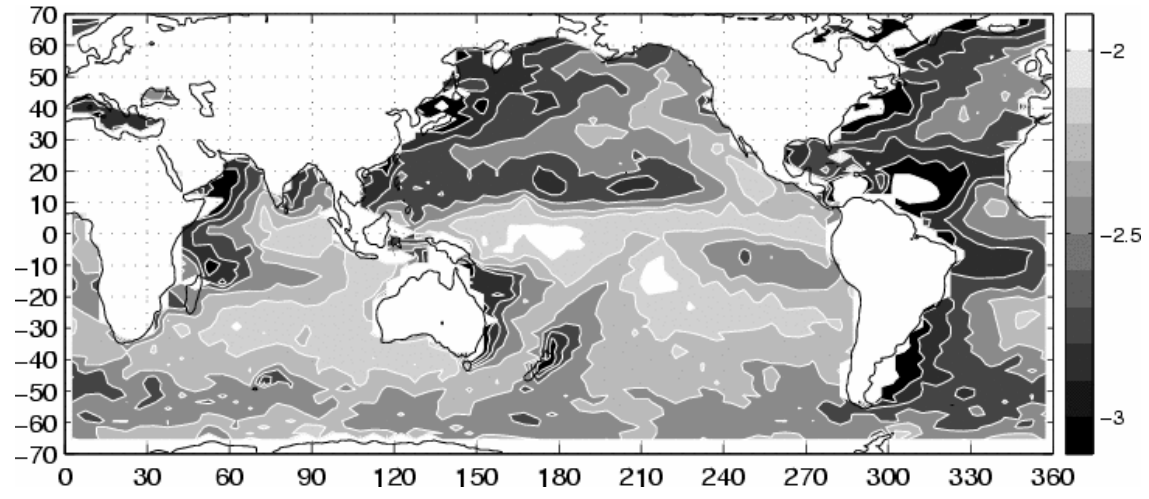

(c)

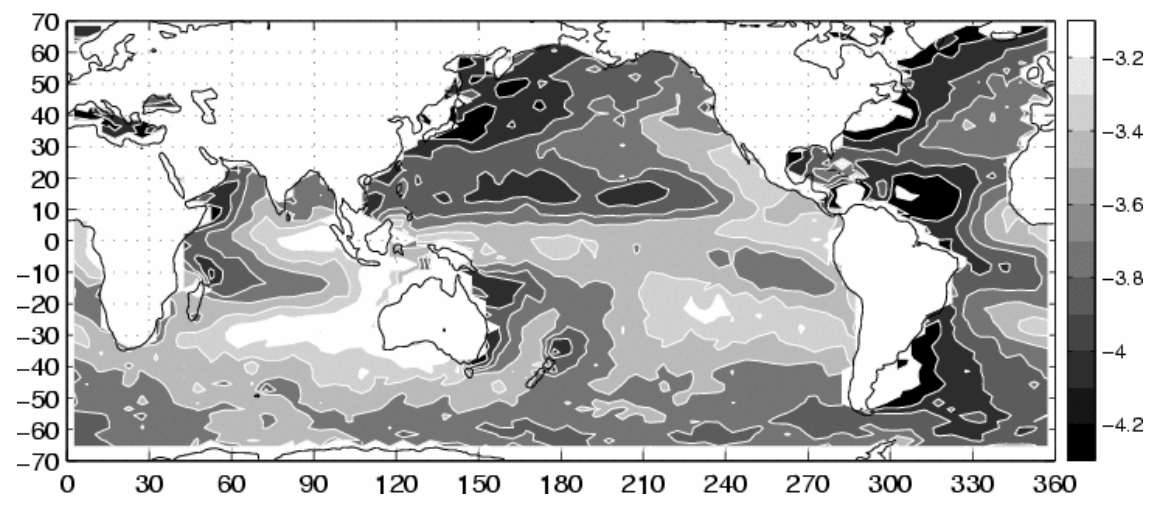

Figure 7: Difference of SSB (cm) mean geographical fields computed between estimates from respectively (a) SSB (SWH, U_alt) with U_alt from MCW algorithm, (b) SSB (SWH, H_swell), (c) SSB (SWH, Tm), and SSB (SWH) models. Note that the colour scale displays the same saturated interval of variation of $1.2 \mathrm{~cm}$ centered on the global mean difference for each map. 
(a)

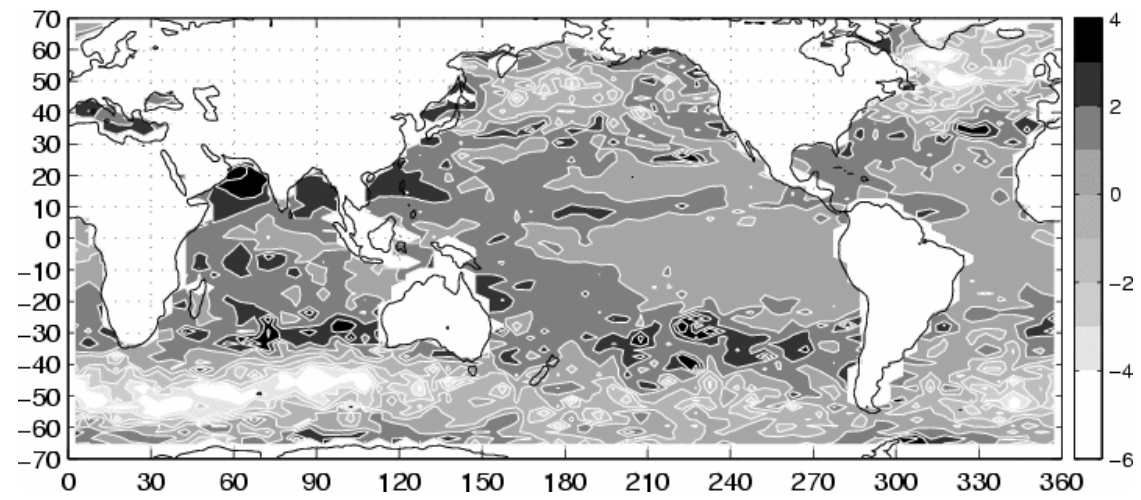

(b)

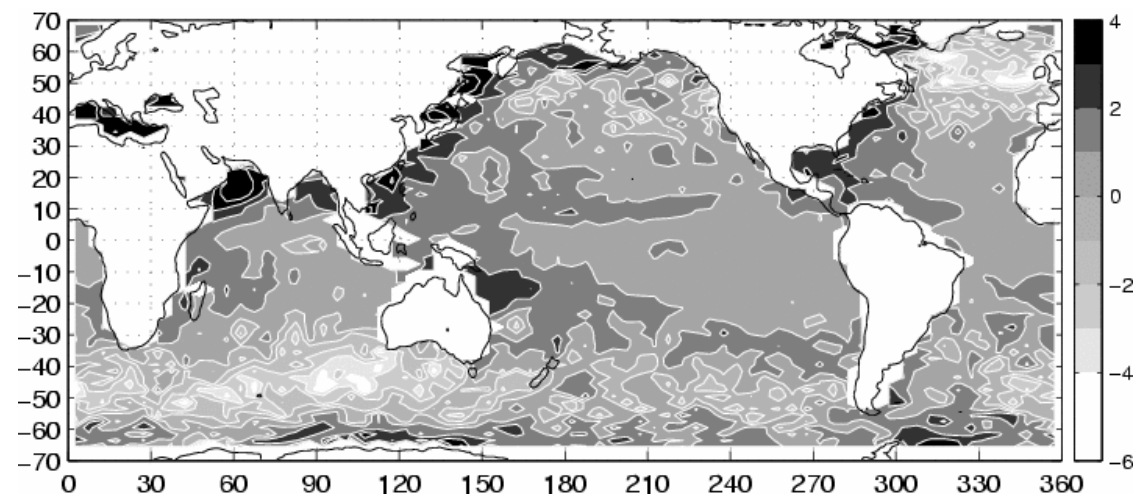

(c)

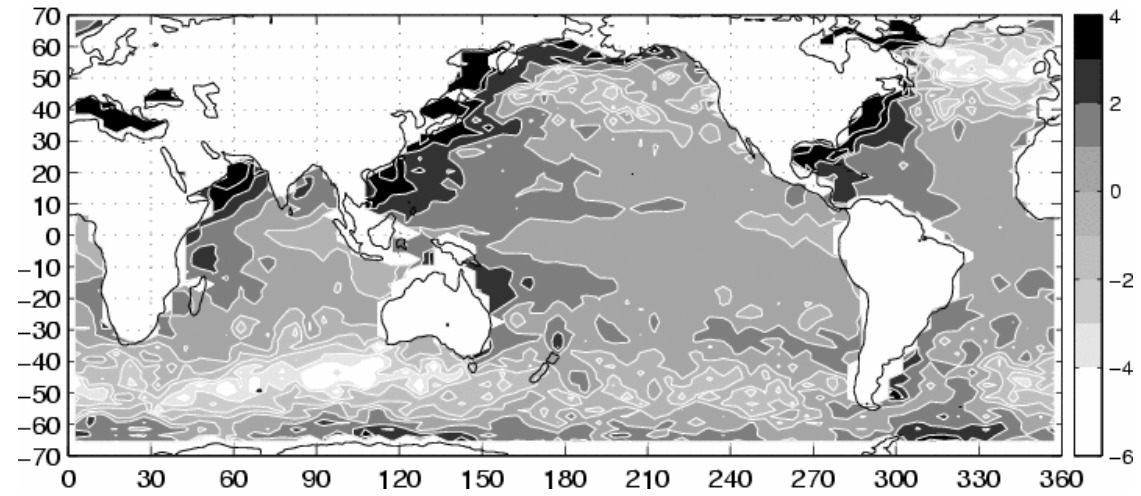

Figure 8: Difference of SSB variability $\left(\mathrm{cm}^{2}\right)$ geographical fields computed with estimates from respectively (a) SSB (SWH, U_alt) with U_alt from MCW algorithm, (b) SSB (SWH, H_swell), (c) SSB (SWH, Tm), and SSB (SWH) models. 